\title{
¿Crisis en la globalización o crisis de la globalización?
}

\author{
Crisis in the globalization or crisis of globalization?
}

JULIETA ZELICOVICH

Doctora en Relaciones Internacionales, Universidad Nacional de Rosario. Investigadora Asistente en Consejo Nacional de Investigaciones Científicas y Técnicas (CONICET). Correo electrónico: julieta.zelicovich@fcpolit.unr.edu.ar

Resumen

Este trabajo tiene como meta presentar un análisis de coyuntura acerca de la llamada "crisis de la globalización". La globalización es entendida como un proceso que tiene lugar en razón de un componente económico-tecnológico, como también político-ideológicoinstitucional; y que periódicamente atraviesa situaciones de crisis. ¿Es la crisis que se manifiesta a partir de 2016 diferente de las anteriores? ¿Qué es aquello que entra en crisis en torno al desarrollo de fenómenos como la elección de Donald Trump, en EEUU-o el Brexit? ¿Cuáles son sus implicancias? A partir de un análisis de la literatura en torno al concepto de "crisis de globalización", y de la contrastación empírica de sus indicadores, se busca dilucidar si se trata de una crisis en la globalización o una crisis de la globalización.

\section{Palabras clave \\ Globalización - Crisis - Orden Internacional - Trump}

\section{Abstract}

The aim of this work is to present an analysis of the context of the socalled "crisis of globalization". Globalization is understood as a process that takes place due to the combination of an economictechnological component, as well as a political-ideological-institutional dimension; and that it periodically becomes in crisis. Is the 2016's crisis different from the previous ones? What is it that it comes into crisis around the development of phenomena such as the election of Donald Trump, in the US, or the Brexit? What are its implications? From an analysis of the literature that deals with the concept of "globalization crisis", and from the empirical testing of its indicators, the aim is to elucidate whether it is a crisis in globalization or a crisis of globalization.

\section{Keywords}

Globalization - Crisis - International Order - Trump 
La globalización es un fenómeno multidimensional, sobre el cual existen múltiples interpretaciones. Mientras que desde las teorías estructuralistas y marxistas se sostiene una lectura de largo plazo, focalizada en el concepto de mundialización el cual se remonta al siglo XIX (Wallerstein, 1997) e incluso, XVI (Wallerstein, 1979)-; las lecturas liberales, hegemónicas dentro de la disciplina, han convergido en la interpretación de que se trata de un fenómeno que tiene sus raíces en el fin de la Segunda Guerra Mundial, y que se manifiesta con vehemencia desde 1990, tras la caída del bloque soviético y hasta la actualidad. En ese marco, la globalización se entiende como el proceso de incremento de la interdependencia entre los Estados, que es impulsado por el comercio internacional, los flujos de capital, el conocimiento y tecnología, y los flujos migratorios; y que tiene lugar en el marco del orden liberal internacional.

Aún situando el inicio de la globalización en 1990, encontramos que éste ha sido un proceso atravesado por numerosas crisis, fundamentalmente económicofinancieras (1995; 1997-1998; 2000; 2008, y posiblemente 2016-18). De hecho, es posible encontrar a la par de estas situaciones un aumento en la producción académica vinculada a la idea de "crisis de globalización". En este trabajo se propone reflexionar en torno a la conceptualización como "crisis de la globalización" de la coyuntura que se desata en 2016 con el voto por la salida de Reino Unido de la Unión Europea -Brexit- y con la elección de Donald Trump como presidente de los EEUU1. Nos preguntamos si en efecto, la globalización ha entrado en crisis; y si se trata de una crisis de la globalización o de una crisis en la globalización.

Asumiendo que la globalización conjuga una base material, con un andamiaje ideológico-institucional que lo acompaña, posibilita y retroalimenta, proponemos una caracterización de la crisis en función de estas dos dimensiones. Sostenemos que, en la coyuntura actual, mientras que la dimensión material ha mantenido su alcance internacional; la dimensión política-ideológica-institucional, inherente a la dinámica de la gobernanza global parece haber entrado en crisis. En el corto plazo se trata más bien de una crisis "en" la globalización que una crisis "de" la globalización; no obstante, una interacción pro-cíclica entre la dimensión ideológica-institucional y material la pueden convertirla a una crisis sistémica.

Mientras que una crisis en la globalización implicaría una crisis que no cuestione los atributos centrales de este orden, y cuyos indicadores presenten una rápida recuperación tras el epicentro del fenómeno; una crisis de la globalización supondría una crisis de carácter sistémico, que cuestione la naturaleza del orden, y que requiera de cambios sustantivos para su resolución. Las dos dimensiones de la globalización pueden actuar como fuerzas que se contienen una a la otra, o pueden entrar ambas en crisis, retroalimentándose entre sí.

\footnotetext{
${ }^{1}$ La crisis de 2016 en adelante suele identificarse con la elección de Trump y el Brexit, aunque las implicancias de ambos fenómenos sean completamente distintas. Mientras que el Brexit solo exhibe un impacto en políticas domésticas, la elección de Trump es tanto una crítica al sistema como la aplicación de políticas domésticas. Véase Primo Braga (2017).
} 
En el próximo apartado se profundizará la definición de globalización y su referencia empírica. Seguidamente se repasará la literatura contemporánea que remite al concepto de crisis de globalización, para luego, en tercer lugar, contrastar esas teorías con los acontecimientos políticos y económicos acaecidos entre fines de 2016 y mediados de 2018.

\section{La conformación de la globalización}

La globalización es, en primer término, un proceso de incremento de la interdependencia entre las economías. Existe una amplia literatura en torno a los factores que le dieron impulso, coincidiendo en la relevancia de dos grandes grupos: por un lado, los avances tecnológicos (menores costos en el transporte y comunicación) y por otro, la cooperación político-económica (convergencia en la liberalización del comercio y apertura de la cuenta de capital).

El desarrollo simultaneo de ambos procesos posibilitó la expansión del comercio global, el establecimiento de redes globales de producción, la conformación de un mercado financiero internacional, acompañado de un importante incremento en el movimiento de personas alrededor del mundo. Estos flujos de factores no fueron distribuidos de manera homogénea en el globo ni al interior de las economías, siendo una de las principales consecuencias de la globalización el incremento en la inequidad del ingreso entre países y al interior de los países (Baldwin, 2016).

El comercio de mercancías pasó de ser el 20,2\% del Producto Bruto Interno (PBI) mundial en 1971, a 51,5\% del PBI mundial en el 2008, año de su pico máximo. Durante todo ese período mantuvo una pendiente positiva, con un segmento especialmente acelerado entre 2001 y 2008. En promedio, desde la Segunda Guerra Mundial el comercio tuvo un crecimiento anual de 1,5 veces el PBI global. Desde 1990 a 2008 la elasticidad fue mayor, alcanzando a crecer al doble que el PBI mundial. Desde 2011 a 2016 se redujo a 1, y en 2016 fue de 0,8, revirtiendo la pendiente anteriormente observada. Por su parte, el comercio de servicios tuvo un incremento sostenido, como rasgo característico de la base material de la globalización, pasando del 7\% del PBI global en 1991 a 12,6\% en 2016.

Los flujos de inversión extranjera directa (IED) por su parte, crecieron abruptamente a partir de 1990, pasando de 0,7\% del PBI en 1991 a 4,35\% en 2000. Siendo un indicador sensible a las crisis, tanto entre 2000-2003 y 2007-09 se presentan caídas importantes, pero manteniéndose en un promedio que triplica al del período de la Guerra Fría (conforme a los datos disponibles en el Banco Mundial). Para el año 2016, la entrada neta de capital en términos mundiales representó el 3\% del PBI.

La fusión de ambos fenómenos, comercio e IED, se reflejó en el establecimiento de cadenas globales de valor (CGV). La fragmentación y deslocalización de los procesos productivos es el rasgo singular del período más dinámico de la globalización. Conforme sostiene Dollar (2017) “Desde 1996 a 2007, el valor agregado en las cadenas globales de valor creció más rápido que otros componentes del PBI (por lo que su participación estaba en aumento). Esto fue especialmente pronunciado en 2002-08, el apogeo de la expansión de CGV" [traducción propia]. Este fenómeno 
tuvo un desarrollo más intensivo en algunas regiones, identificándose tres nodos, que actuaron como ejes de los intercambios comerciales y que dieron lugar a la conformación de tres grandes "fabricas": la fábrica Asia, la fábrica Europa y la Fábrica norteamericana. En este aspecto, la globalización fue heterogénea en términos regionales.

Los movimientos financieros se acentuaron durante el período de la globalización. La inversión en cartera ${ }^{2}$ comenzó a crecer de manera dinámica a partir de la década del 90, pasando de un total de flujos mundiales por 85 mil millones de dólares en 1991 a 647 mil millones en el 2000; 905 mil millones en 2005, y superando el billón de dólares en 2014, aunque con fuertes contracciones en 2001, 2008, 2011 y nuevamente 2016. El nivel de deuda global ha ido incrementándose de manera sostenida desde la Segunda Guerra Mundial e inclusive, ha superado actualmente a su pico anterior del año 2009. Conforme a Mbaye, Moreno Badia, y Chae (2018) "almost a decade after the collapse of Lehman Brothers, global debt, at \$164 trillionor about 225 percent of global GDP_has reached new record highs (April 2018 Fiscal Monitor)". EEUU, China y Japón explican más del $50 \%$ de esta deuda; aunque desde la crisis financiera de 2008 ha sido el sector privado de las economías emergentes el que más se expandió en el proceso de endeudamiento.

En cuanto al desarrollo tecnológico, Baldwin (2016) indica como la capacidad de transmisión y almacenamiento se duplicó anualmente durante el tramo analizado, en tanto que la capacidad de las computadoras ha crecido de manera exponencial. Para el mencionado autor, otro elemento tecnológico relevante es el desarrollo y expansión de los fletes aéreos dentro de las logísticas del comercio y las cadenas de valor.

Con la extensión de estas redes materiales se fue consolidando una mayor interdependencia de las economías en términos globales: una dependencia mutua entre los estados que generó efectos mutuos de las acciones. Así, las decisiones económicas de un estado suelen repercutir en el resto 3 .

Esta mayor vinculación entre las economías y sus sociedades se vio posibilitada por una convergencia en la dimensión política-ideológica-institucional, acompañada de un liderazgo dentro del orden internacional, que condujo a la consolidación de ciertos marcos de cooperación, plasmados en organismos internacionales. Estos marcos dieron sustento y encausaron la globalización.

Conforme a Ikenberry (2017) cinco serían los componentes de este orden liberal asociado a la globalización:

En primer término, apertura económica. Tanto en lo relativo al comercio como a la política financiera y monetaria, el orden liberal marcó una priorización del Mercado por sobre el Estado, cuyo tope fue el límite de la estabilidad socio-económica

${ }^{2}$ Los valores en cartera incluyen la entrada neta de valores de renta variable, que no sean los registrados como inversiones directas e incluyen acciones, valores, recibos de depósito (estadounidenses o mundiales) y compras directas de acciones en los mercados de valores locales por parte de inversiones extranjeras.

${ }^{3}$ Por ejemplo, tal como establecen lacoviello y Navarro (2018) "In response to a U.S. monetary tightening, GDP in foreign economies drops about as much as it does in the United States, with a larger decline in emerging economies than in advanced economies". 
doméstica (lo que Ruggie denominó "liberalismo enmarcado" o "embedded liberalism"). Los estados han convergido en un conjunto de políticas de desregulación de la economía preservando cierto grado de intervención en razón de lo que había sido la estructura del Estado de Bienestar: "multilateralism was joined by collaboration to assure domestic economic growth and social security as early as the Atlantic Charter" (Ruggie, 1992).

La segunda característica del orden liberal ha sido la consolidación de instituciones multilaterales. El Fondo Monetario Internacional (FMI), el Banco Mundial, y muy posteriormente la Organización Mundial de Comercio (OMC), en reemplazo del Acuerdo General de Comercio y Aranceles (GATT), constituyen la arquitectura institucional multilateral que le dio forma al desarrollo de la globalización económica bajo el liderazgo norteamericano. Los principios de no discriminación, indivisibilidad y reciprocidad difusa han orientado estas instituciones (Ruggie, 1992). Esta última resulta una pieza clave en la extensión de la cooperación. Conforme la define Keohane (1986) la reciprocidad difusa, opuesta a la reciprocidad específica, orienta a la conducta del Estado no en función de una recompensa inmediata de actores específicos, sino en razón de la obtención de resultados globales satisfactorios para todo el grupo del que forma parte, como un todo. El ejemplo es el principio de la cláusula de "Nación Más Favorecida", según el cual, cualquier favor o privilegio que un Estado le da a otro, debe ser inmediatamente extendido a todos los miembros del grupo. De esta forma, los países involucrados esperan que el acuerdo produzca una "equivalencia aproximada de los beneficios en el agregado y en el tiempo" (Ruggie, 1992: 571).

Finalmente, el orden liberal se ha sostenido en base a la cooperación en materia de seguridad, a la solidad democrática y al ideario internacionalista. Estos se plasmaron fundamentalmente en el accionar de Naciones Unidas y en el liderazgo de los EEUU, que proveyeron los incentivos necesarios para que estos principios se concretaran. El orden liberal asociado a la globalización ha sido un orden "americano". Principalmente a través del GATT, aunque no de manera exclusiva mediante el mismo, EEUU logró orientar a otros Estados hacia conductas cooperativas, dando lugar a un bien público. EEUU fue el protagonista central de todos estos movimientos durante la década del 90 y hasta mitad de los 2000, cuando se puso de manifiesto el ascenso de economías emergentes y, en particular, de China; comenzando entonces el desafío tácito- al orden imperante.

\section{La delimitación teórica de la crisis de globalización contemporánea.}

Este orden liberal asociado al desarrollo de la globalización había sufrido solo ajustes menores, en particular en el paso de una globalización "enmarcada" a una "hiperglobalización", en los 904, pero no había sido cuestionado de manera sustantiva. El voto en el referéndum británico en favor del "Brexit" y la elección de Donald Trump en los EEUU, han sido considerados un quiebre en dicha trayectoria. Ambos sucesos emergen como símbolos de la manifestación de una nueva crisis de la globalización.

\footnotetext{
${ }^{4}$ Véase Subramanian (2013); UNCTAD (2017).
} 
En la literatura reciente han aparecido tres conceptualizaciones para referir al fenómeno: "Crisis de globalización" (crisis of globalization o globalization's crisis), "contragolpe a la globalización" (Backlash against globalization); y crisis del orden liberal.

Los diagnósticos de causas y mecanismos de la crisis son convergentes entre los enfoques. Conforme a esta literatura, en torno al año 2016, se produjo un crecimiento del nacionalismo y del populismo en diferentes lugares del mundo (Hale y Held, 2018) acompañado de una creciente polarización y movilización en torno al desempeño de las instituciones internacionales, no cuestionadas anteriormente (Zurn, 2018). En términos de orden internacional, los rasgo en los que todos acuerdan son un menor liderazgo de EEUU, la expansión de lecturas mercantilistas y lecturas del escenario internacional como un juego de suma cero (Hays, 2017).

Estas transformaciones habrían tenido lugar debido a una serie de "promesas incumplidas" en torno a los desafíos de la globalización (Hale y Held, 2018), las cuales se habrían originado en primer término por un cambio en la dinámica del orden liberal. Conforme establece Ikenberry (2017), entre otros, "With the collapse of the Soviet Union, the liberal order expanded across the globe, and sowed the seeds for today's crisis: it lost its embedded, protective qualities and was increasingly seen as a neoliberal project aimed at facilitating the transactions of globetrotting capitalists." 5 .

Una de las consecuencias más severas de esta transformación en las políticas hacia la globalización fue la profundización en las inequidades en torno a la distribución del ingreso y salida de la pobreza ${ }^{6}$. Las clases trabajadoras de los países desarrollados vieron reducidos sus empleos por el proceso de internacionalización y deslocalización de las empresas y de la revolución tecnológica. En efecto, los efectos distributivos de la Globalización son señalados en la literatura como el eje central de esta crisis (Fukuyama, 2016; Baldwin, 2016).

El otro componente necesario para el desarrollo de la crisis en cuestión es la canalización política de esos descontentos y reclamos, a través de alternativas electorales no disponibles en períodos anteriores". Conforme señala Hays "This is a fundamental change in the domestic political landscape that has profound implications for the global economy" (Hays, 2017). La respuesta de esta "nueva clase política" frente a la crisis externa no ha sido la cooperación internacional, sino más bien la priorización del ámbito nacional, dando lugar al proteccionismo comercial y a la "balkanización" de la regulación financiera (Boughton, 2017). La imposibilidad de arribar a un mecanismo consensuado de reacción a la crisis de 2008 (UNCTAD, 2017) se encuentra en los cimientos de esta nueva crisis, ahora con epicentro político.

En cuanto a las implicancias y alcances de la crisis, las lecturas que se enfocan en el conflicto ideológico en torno a la globalización plantean que se trata de una crisis

\footnotetext{
${ }^{5}$ Casi quince años antes de que aconteciera esta "crisis de la globalización" Stiglitz sostenía que "the ideas and intentions behind the creation of the international economic institutions were good ones, yet they gradually evolved over the years to become something very different" (Stiglitz, 2002: 16).

${ }^{6}$ En "Globalization and its discontents" Stiglitz señala que para el año 2002 mientras que el PBI mundial había crecido a una tasa de $2,5 \%$ anual, la pobreza no había disminuido, sino que había aumentado a 100 millones.

${ }^{7}$ Por ejemplo, el Movimiento Cinque Stelle y Liga Nord en Italia.
} 
terminal, propia de los agotamientos cíclicos de los diversos órdenes económicos internacionales de la historia (Hays, 2017). Los enfoques que ponen el foco en los elementos constitutivos del orden liberal plantean que la crisis es severa, aunque no terminal, y que resultaría necesario recuperar los valores de "seguridad cooperativa", en torno a la democracia y bienestar económico; así como la emergencia de nuevos liderazgos (Ikenberry, 2017). Otros plantean un posible orden de "post-globalización" en el que "la globalización sigue en desarrollo, pero con nuevas características" (Serbin, 2018). Tales enfoques, próximos a este estudio, sostienen que se trata de una crisis de gobernanza global antes que de globalización. Por su parte, trabajos como los de Hale y Held (2018), sostienen que a pesar del carácter cada vez más estructural del bloqueo existente en la globalización, pueden identificarse vías para un sostenimiento del orden multilateral a través del fortalecimiento de instituciones, y el involucramiento de actores no-estatales.

\section{Aproximación empírica a la crisis de globalización contemporánea}

La literatura referida acierta en el diagnóstico de las causas del problema, tiende a sostener que este es de carácter sistémico -crisis de la globalización-, pero se muestra optimista en cuanto a que el deterioro no es aún irreversible. En este apartado argumentaremos que, en el corto plazo, no se trata de una crisis de la globalización, sino de una crisis en la globalización. Para ello nos basamos en dos argumentos que tienden a ser relegados en la literatura anteriormente mencionada. El primero es la tendencia contracíclica que presenta la dimensión económicacomercial; el otro, es el sostenimiento de lazos cooperativos en las relaciones del resto de los países del mundo, cuando éstas no involucran a EEUU.

Mientras que la dimensión político-ideológica ha sufrido alteraciones, la dimensión económica-comercial ha mantenido su nivel de expansión y de interdependencia. Se registran ajustes en la dimensión económica-comercial, pero estos anteceden a la crisis política y son de más largo plazo. La globalización, en el siglo $\mathrm{XXI}$, se encuentra en un proceso de transformaciones, pero no es ésta su crisis terminal; la coyuntura actual no se trata de una crisis sistémica, sino de una crisis de la dimensión política-ideológica-institucional.

Kotz (2009) plantea la crisis sistémica como una crisis que solo se puede resolver a través de una importante restructuración del sistema. En modo contrario, una crisis no sistémica, se resuelve con mecanismos existentes dentro de las instituciones (planes de estímulo, por ejemplo) y algunas nuevas regulaciones pero que no cambian lo sustancial del orden.

Una limitación de este enfoque es que no existe, sin embargo, un criterio de interpretación para establecer un límite acerca de qué es lo sustancial del orden, en tanto que en la idea de "crisis" subyace un amplio espectro de situaciones. Para Kotz (2009), algunos ejemplos de crisis sistémica fueron la crisis de 1929, que derivó en el agotamiento del modelo liberal, la segunda guerra mundial y su reemplazo con el orden de Bretton Woods; y la crisis de los 70, que derivó en el agotamiento del modelo del estado de bienestar y la reforma sustantiva del orden del Bretton Woods a partir de la remoción del tipo de cambio fijo en torno a 75 dólares la onza de oro, y que 
terminó dando paso a la adopción de un régimen neoliberal en la globalización. En cambio para Ruggie, tanto los acuerdos de Jamaica (orden monetario) como la Ronda Tokio (comercio) implicaron una gran transformación del orden, pero que no supusieron una crisis de su naturaleza, sino que fue un cambio orientado por las propias normas del sistema (norm governed change).

En el siglo XXI, la primera crisis acaecida dentro de la globalización fue la crisis financiera internacional de los años 2007-2008, cuyo epicentro fueron los países desarrollados. A diferencia del proceso actual, esta crisis fue principalmente material: financiera y comercial, y no puso en cuestión la reproducibilidad de la propia globalización. Por el contrario, la salida de la crisis, asimismo, condujo a un fortalecimiento de las estructuras existentes de gobernanza (Garrido, et. al., 2016; Woods, 2010). Las reacciones posteriores al 2008 llevaron a acentuar la globalización liberal. Por ejemplo, se produjo una

Mejora de la gobernanza y legitimidad con reequilibrio del poder de voto y realineamiento del Directorio en favor de economías emergentes. Se triplican los recursos de naturaleza permanente, se flexibiliza la política de préstamo de resolución de crisis (condicionalidad más realista y mayor acceso a recursos), y se crean nuevos instrumentos precautorios. Nuevas formas de trabajar en coordinación con terceros (troika en Europa). Aumento histórico del volumen de préstamos, con una media anual de $74 \mathrm{~mm}$ de DEG (frente a $13 \mathrm{~mm}$ en 19902008) y el máximo anual de más de $140 \mathrm{~mm}$ en 2011 (el máximo anual en años anteriores no llegaba a $40 \mathrm{~mm}$ ). (Garrido, et. al., 2016)

La dimensión política exhibió un ajuste en conformidad a la nueva distribución de poder: el foro de concertación principal de políticas pasó del G7 al G20. Pero su efecto fue el de expandir las convergencias ideológicas en torno a la globalización. Tal como afirman Cooper y Poulot (2015) la capacidad de transformación del G20 en relación al $\mathrm{G} 7$ ha sido limitada y, de hecho, en el plano de los procesos diplomáticos el G20 replicó las mismas prácticas de refuerzo del orden oligárquico que el G7 en los mecanismos de creación de reglas, en el uso de criterios arbitrarios para la inclusión de miembros y en la reproducción de mecanismo de cooptación de disciplinamiento. En suma, la crisis de 2008 fue de naturaleza eminentemente económica y no se produjeron crisis sustantivas en la dimensión política por parte de los países centrales. Por el contrario, esta última actuó como fuerza contracíclica a las tendencias de la dimensión económica

La crisis de 2016-2018 tiene foco prioritario, en cambio, en la dimensión política-ideológica-institucional. La existencia de disonancias en la dimensión política dentro de la globalización no es nueva, sin embargo, se presentan dos diferencias con relación a críticas de períodos previos. La primera deriva de la naturaleza del orden internacional y de la distribución del poder global. Mientras que las críticas políticas de los países en desarrollo -cuyo registro es anterior, siendo un antecedente ineludible el discurso en torno al Nuevo Orden Económico Internacional de la década 
del 70- no tienen impacto sobre la globalización y se dan en el marco del "funcionamiento normal" de la dimensión política; las críticas de los países desarrollados, impactan de manera sustantiva. Se trata de una suerte de "geopolítica del descontento". EEUU fue durante toda la segunda mitad del siglo XX el constructor y garante del orden liberal que dio forma a la globalización y de allí que su posicionamiento frente al mismo resulte de particular importancia.

La otra diferencia es que mientras que en las crisis previas las críticas hacia el orden internacional eran manifestadas dentro de las instituciones internacionales esto es del Estado hacia afuera- ahora dicho reclamo es canalizado en políticas nacionales que repercuten en el funcionamiento de la globalización. No solo en la emergencia de alternativas electorales contra-globalización otrora inexistentes, sino también en la implementación de políticas domésticas que obran en sentido inverso al de la globalización. La dimensión internacional -la situación de la globalizaciónimpacta en la política nacional -en sus alternativas electorales-; y éstas vuelven a impactar en la dimensión internacional. Como sostiene Gourevitch "The international system is not only a consequence of domestic politics and structures but a cause of them." (Gourevitch, 1978: 911)

Así, el proceso en curso tiene epicentro dentro de la dimensión políticaideológica-institucional: la asunción de un gobierno en los EEUU que se presenta contrario a la globalización y que implementa medidas que desafían el orden establecido. Las políticas proteccionistas del comercio ${ }^{8}$ son el instrumento de las expresiones de la crítica y cuestionamiento hacia la globalización por parte del gobierno de Trump, las que se expresan en el discurso del American First.

Se ponen en cuestión, en primer término, la idea de liberalización; en segundo lugar, la idea de reciprocidad difusa; y en tercer lugar las instituciones internacionales predominantes o reglas del juego global vigentes; tres componentes claves del orden liberal, según expusimos anteriormente.

El primer punto de cuestionamiento, sobre la apertura económica, se centra en un enfoque mercantilista de la economía. Esta es lógica filosófica-económica que subyace al gobierno que asume en 2016. Se trata de una escuela de pensamiento que tuvo su auge en el período del siglo XVI al XVIII y que asocia el superávit de la balanza comercial con el poder y seguridad del Estado. El comercio se convierte así en un juego de suma cero, donde las ganancias de uno son pérdidas para el otro. Los sectores son considerados estratégicos en función de su aporte a la seguridad nacional y no debido a ventajas comparativas y competitivas -como sostienen otros enfoques.

Desde esta perspectiva, el principal problema de los EEUU en la globalización es su déficit comercial internacional (el cual rondaba los 796 mil millones de dólares

\footnotetext{
${ }^{8}$ En términos generales cabe señalar que la política comercial adquirió relevancia electoral. Según señala VanGrasstek (2017) "The profile on this topic rose in the early stages of the debate over globalization, but declined as the political center of gravity among corporate leaders and other elites shifted towards acceptance of open markets". Así, la atención presidencial y la presentación de leyes al congreso por parte de G. W. Bush y Obama fue mucho menor que la de Bush y Clinton. Sin embargo, Trump encontró en ese punto una brecha que aprovechó electoralmente (VanGrasstek, 2017).
} 
al asumir Trump) y la manera de contrarrestarlo es a través de medidas de restricción a las importaciones. En consonancia, desde enero de 2017 a mayo de 2018 , EEUU implementó 200 medidas restrictivas del comercio ${ }^{9}$. El punto crítico de estas medidas no ha sido tanto su cantidad ni el comercio abarcado (a mayo no habían superado el pico de 2011) sino la naturaleza de las mismas, en confrontación y desafío con los compromisos internacionales en la OMC. A lo cual hay que sumar la relevancia que se le otorgó al discurso del poder ejecutivo, tanto hacia el interior del país como hacia fuera de los EEUU. Se combinaron medidas de salvaguardia (sobre lavarropas y paneles solares), suba de aranceles generales (acero y aluminio ${ }^{10}$ ) bajo la pretensión de ser excepciones por razones de seguridad nacional, así como aranceles para socios específicos (un listado de diversos productos por un volumen de 34 billones de dólares, en julio 2018, contra china) por pretendidas situaciones de comercio "injusto", especialmente en lo correspondiente a transferencia tecnológica y propiedad intelectual (sección 301 de la Trade Act de 1974).

El segundo eje de desafío al orden existente provino del cambio de una reciprocidad difusa a una reciprocidad específica. EEUU propuso una revisión de los acuerdos de comercio existentes, que "re balancearan" las relaciones comerciales de socios claves con EEUU, y el alejamiento del foro multilateral. Conforme se explicita en el documento "Trade Policy Agenda and Annual Report of the President of the United States on the Trade Agreements Program" del año 2017:

The overarching purpose of our trade policy - the guiding principle behind all of our actions in this key area - will be to expand trade in a way that is freer and fairer for all Americans (...) As a general matter, we believe that these goals can be best accomplished by focusing on bilateral negotiations rather than multilateral negotiations - and by renegotiating and revising trade agreements when our goals are not being met.

Ello se instrumentó en el retiro de EEUU de las negociaciones del Acuerdo Transpacífico (TPP), en enero de 2016; en las re-negociaciones del Tratado de Libre Comercio de América del Norte (TLCAN o NAFTA), las cuales comenzaron en agosto 2017; y la revisión del acuerdo EEUU-Corea del Sur, entre octubre 2017 y mayo 2018, y en el discurso en la Ronda Doha. Conforme al Departamento de Comercio de los EEUU, el NAFTA habría permitido a las empresas norteamericanas aprovecharse de los bajos salarios mexicanos a costa de los trabajadores de EEUU; y si bien el NAFTA tenía previsiones en derechos laborales y ambientales, estos no resultaban lo suficientemente sólidos. De allí la necesidad de elevar el tratado a los "estándares del

\footnotetext{
${ }^{9}$ Las restricciones sobre las importaciones fueron acompañadas por la sanción de una reforma fiscal que hizo hincapié en la internacionalización de las empresas. Esta apunta a "favorecer el crecimiento, la creación de empleo y la inversión doméstica, desincentivando la deslocalización de la actividad y el beneficio de las empresas multinacionales en el extranjero" (Álvarez García, 2018)

${ }^{10}$ Los principales afectados por esta medida fueron socios tradicionales de EEUU, como Canadá. Las importaciones chinas ya estaban cubiertas por una medida antidumping.
} 
siglo XXI" y "re balancear el NAFTA" para que no sea un incentivo a las empresas norteamericanas para la radicación de sus inversiones fuera del territorio. (USTR 2018). Por su parte, en el acuerdo con Corea del Sur, la meta de EEUU era el balance en el sector de vehículos y autopartes y el incremento en la vigilancia y cumplimiento de regulaciones pendientes del Acuerdo de Libre Comercio bilateral, conocido como KORUS, por parte de Corea del Sur.

En cuanto al ámbito multilateral, durante la Conferencia Ministerial de Buenos Aires, el discurso del embajador Lighthizer, representante de EEUU, hizo tres críticas sustantivas hacia la OMC como foro para negociaciones entre sus miembros, una de las cuales se relaciona de manera estrecha con la concepción de la reciprocidad específica. Lighthizer remarcó la situación respecto de los países en desarrollo y cómo "five of the six richest countries in the world presently claim developing country status" (Lighthizer, 2017), lo que los habilita a recibir un trato especial y diferenciado -que resulta incompatible con una noción de reciprocidad específica. Las otras dos críticas apuntan al creciente rol del órgano de solución de diferencias de la OMC ${ }^{11}$, lo cual se contrapone con la concepción respecto de la supranacionalidad e internacionalismo en el gobierno de Trump; y respecto del cumplimiento de las normas de la OMC en terceros países, lo cual lleva a introducir el concepto de "comercio justo" dentro de la política comercial externa de Trump.

En efecto, en el marco del discurso del "America First", se priorizó el concepto de "fair trade", o comercio "justo", el cual entró en tensión con "libre" comercio, o comercio basado en normas. Fair trade remite a una reevaluación de las políticas de los socios comerciales de EEUU, en razón de una priorización de las regulaciones domésticas norteamericanas por sobre los compromisos internacionales. En consonancia se produjo un incremento de la función de vigilancia que ya venía llevando adelante la política comercial norteamericana. Esta lectura sustanció también una política de crítica y erosión al Órgano de Solución de Diferencias de la OMC.

Los tres puntos presentados hasta aquí plantean un cuestionamiento profundo a la naturaleza de la dimensión política-ideológica-institucional. Sin embargo, su alcance, en términos de crisis sistémica, depende de dos puntos: Hasta qué punto estos cuestionamientos por parte del gobierno de Trump han sido replicados por otros actores en el marco de la Globalización; y el segundo, cómo se ha reflejado ello en la dimensión económica.

Respecto de la cooperación en materia comercial y apoyo al multilateralismo, no se observa una retracción equivalente a la de EEUU en otros países. Por ejemplo, ante la retirada de EEUU del TPP, en el mes de enero de 2017, los demás signatarios del acuerdo convinieron continuar con la iniciativa, y en el mes de noviembre 2017, en el marco de la reunión de la APEC, retomaron el proceso de cooperación

\footnotetext{
${ }^{11}$ A raíz de ello EEUU procedió a una política de veto sistemático sobre el nombramiento de todos los posibles candidatos para integrar el Órgano de Solución de Diferencias de la OMC. Véase Foltea (2018).
} 
Perspectivas Revista de Ciencias Sociales - ISSN 2525-1112|Año 3 No. 6 Julio-Diciembre 2018, pp. 42-59

económica, derivando en marzo 2018 en la firma del Tratado Integral y Progresista de Asociación transpacífico, CPTPP o TPP $11^{12}$.

Europa, por su parte, vio en la crisis desatada por Trump una veta para fortalecer un liderazgo blando en torno al multilateralismo. En un documento oficial se sostuvo:

Challenges ahead, particularly in a multipolar world, will require that we continue and strengthen economic policy coordination to ensure strong, sustainable, balanced and inclusive growth. Multilateral cooperation with our global partners remains our preferred approach. To that end, we should contribute to the strength and reform of multilateral institutions to make them fairer and more effective so that they remain a part of the solution. Yet in an increasingly contested global order, we should also be ready to push ahead through cooperation with smaller coalitions, while leaving the door open for others to join when ready. Likewise, non-state actors such as international or non-governmental organisations should be appropriately involved. (European Commission, 2017)

En tal marco, en el período bajo estudio, si bien las negociaciones por el acuerdo Transatlántico (TTIP) quedaron congelado, Europa logró avanzar en la firma y ratificación del acuerdo con Canadá (CETA); la ampliación del acuerdo MéxicoUnión Europea y la firma de un importante acuerdo de comercio e inversiones con Japón; en tanto ha profundizado las negociaciones comerciales con MERCOSUR y Singapur

Al igual que en el caso de Europa, China, en la cumbre de Davos de 2017, reafirmó su creciente involucramiento en las cuestiones internacionales y un mayor compromiso con el libre comercio, al cual lo consideró "to be a right strategic choice". En la esfera de los acuerdos preferenciales, China continúa negociando el RCEP, e impulsó importantes acuerdos comerciales como el de China-Japón-Corea; en tanto viene implementando una ambiciosa iniciativa de inversiones: la nueva Ruta de la Seda, la cual involucra a más de 69 países y organizaciones internacionales, y proyecta un volumen de 800 billones de dólares en infraestructura y servicios complementarios, de los cuales ya ha ejecutado 60 billones.

En contraste, las organizaciones multilaterales y foros de concertación no lograron dar una respuesta concreta a los cambios impulsados por EEUU. La OMC, si bien logró la puesta en vigor del Acuerdo de Facilitación del Comercio ${ }^{13}$, permaneció estancada en su función de negociaciones. La Conferencia Ministerial de Buenos Aires no logró aportar ningún elemento que pudiera revertir la crisis de la dimensión política de la globalización, en tanto que el Órgano de Apelaciones presenta dificultades de funcionamiento ante los reiterados vetos de EEUU al nombramiento de

\footnotetext{
${ }^{12}$ Para ello decidieron incorporar 20 suspensiones a los compromisos del tratado original, en aspectos tales como inversión, propiedad intelectual, productos farmacéuticos, servicios postales, servicios de internet y compras gubernamentales.

${ }^{13}$ Para fines de 2017, 126 países ya habían ratificado el acuerdo.
} 
árbitros. La cumbre del G20 en Alemania, en 2017, no logra alcanzar una declaración final sobre el área del comercio internacional; y la cumbre del G7 en Canadá en 2018, culminó en un fracaso en materia de cooperación comercial internacional.

En cuanto a las reacciones a las medidas proteccionistas de los EEUU, se han articulado tres tipos de reacciones: a) el establecimiento de demandas dentro del Órgano de Solución de Diferencias de la OMC ${ }^{14}$; b) la negociación bilateral15; y c) la retaliación o contramedidas. Una cuarta opción ha sido la denuncia en los foros multilaterales y plurilaterales como el G20 y el G7, llevando al desarrollo de un clima de confrontación dentro de estas cumbres, que en su mayoría fracasaron, como se expuso anteriormente.

Mientras que las demandas en la OMC y la negociación constituyen vías que mantienen la lógica cooperativa, la retaliación posibilita un escenario de "guerra comercial", con reacciones y contra-reacciones en alza. De acentuarse este es el proceso que mayores potencialidades tiene de traducir una crisis de la dimensión política en una crisis sistémica de la globalización con afectación sobre la dimensión material. Sobre esta línea se han concentrado las acciones de China, México, Canadá, la Unión Europea, y recientemente, Rusia. Los vínculos afectados han sido, no obstante, limitados a la relación con EEUU, manteniéndose la vía cooperativa con terceros Estados.

Los impactos sobre la dimensión económica han sido hasta el momento limitados, lo que, junto a la persistencia de mecanismos de cooperación, hace pensar que se trata de una crisis en la globalización antes que una crisis de la globalización. No obstante, debe señalarse que la dimensión material: aún registra rastros de la crisis del decenio anterior; y exhibe algunas señales de ajustes dentro de la propia globalización.

Uno de los cambios más significativos del período entre la crisis de 2008 y la de 2016-18 ha sido la pérdida de capacidad del comercio para impulsar el PBI mundial. En el tramo de 2011 a 2016 el comercio perdió 8 puntos porcentuales sobre el PBI global, presentando una pendiente negativa (datos banco mundial). Ello para muchos ha sido considerado como un posible cambio en la naturaleza de la globalización si se la compara con las décadas de los 90's y 2000's. Tras la crisis financiera, el comercio pasó a crecer al mismo ritmo que el PBI mundial, o incluso a uno menor. Sin embargo, el escenario de crisis política de 2016-18 no parece haber profundizado esta tendencia. Si bien el comercio continúa evolucionando a un ritmo menor que el de la década previa a la crisis de 2008, desde 2017 presenta una pendiente positiva, alcanzando valores superiores: en 2017 el crecimiento del comercio mundial fue de 4,7\%. Para el 2018 y 2019 la OMC estima un crecimiento del comercio mundial del $4.4 \%$. Aún con la incertidumbre que deriva de la particular

\footnotetext{
${ }^{14}$ Corea presentó una demanda por las salvaguardas en lavarropas y paneles de energía solar; México, Canadá, India, Unión Europea, y China, solicitaron, cada uno de forma separada el establecimiento de un Grupo Especial para dirimir acerca de las medidas implementadas al Aluminio y al Acero. EEUU y la UE presentaron a su vez demandas contra China, el primero por la protección de la propiedad intelectual y la segunda por la transferencia en tecnología.

${ }^{15}$ En los casos de Corea, Argentina, Australia, Brasil y China. Aunque en este último caso las negociaciones fracasaron debido a las medidas adoptadas por EEUU tras los acuerdos.
} 
situación que acontece en la dimensión política, las exportaciones e importaciones mundiales correspondientes al mes de marzo de 2018 han sido mayores a las de 2017, y si bien en abril se presenta una contracción en los montos exportados, estos siguen siendo mayores al promedio del trimestre (gráfico 1). Y, del mismo modo, las transacciones de EEUU muestran para los meses de 2018 mayores valores que los de 2017. En cuanto a cómo se distribuye ese comercio, durante los dos últimos años no se registraron cambios sustantivos en los porcentajes de participación de los diferentes países.

La cobertura de las medidas que restringen a las importaciones del período octubre 2017 a mayo 2018 alcanzó los 74.1 billones de dólares, lo que implicó una participación de $0.43 \%$ del total de las importaciones del G20 y del $0.33 \%$ de las importaciones mundiales. Estos montos son mayores que los de todo el 2016, pero equivalentes al período octubre 2015 mayor 2016. Por su parte, consistente con las observaciones en torno a la cooperación internacional del resto de los países, la cobertura de las medidas destinadas a facilitar las importaciones (US\$82.7 billones) ha superado a las medidas restrictivas (OMC, 2018).

\section{Gráfico 1}

\section{Evolución mensual de las exportaciones mundiales} (en billones de dólares)
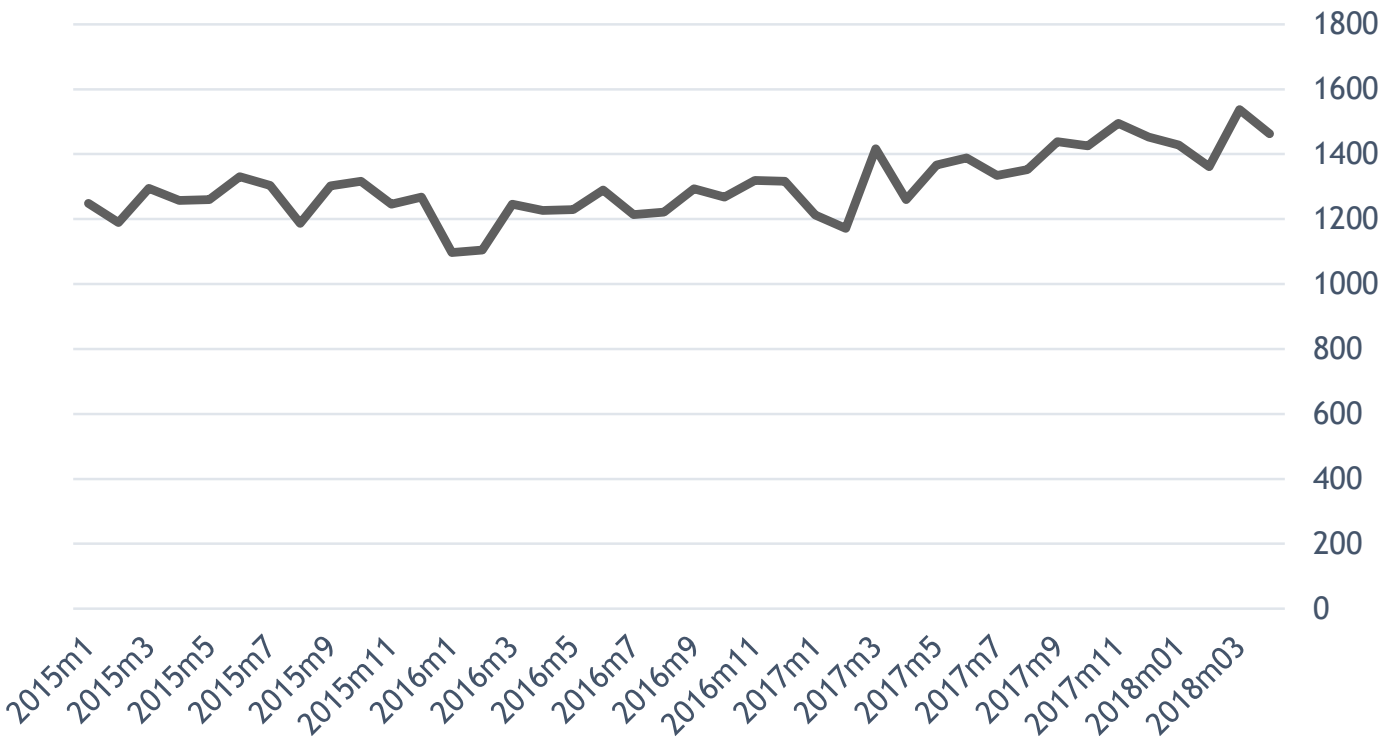

Fuente: Elaboración Propia en Base a Estadísticas de Corto Plazo de la OMC.

El comercio, no obstante, crece a un ritmo más bajo en relación al $\mathrm{PBI}$ de lo que lo hacía en el auge de la globalización. Este cambio se adjudica al dinamismo de las cadenas globales de valor y antecede a la crisis política de la globalización. Desde la crisis financiera internacional las CGV dejaron de expandirse y mantuvieron estables sus niveles de intercambio. Según gran parte de la literatura, esto se 
explicaría por la reorientación del modelo económico chino y su mayor énfasis en el mercado doméstico. Cabe señalar que los últimos datos disponibles son del año 2015 , no siendo aún viable profundizar el análisis de los efectos de la crisis política de la globalización sobre los resultados de las cadenas productivas. No obstante, la inversión extranjera directa, la cual expresa la deslocalización de la producción y la integración en "fabricas mundiales" -un elemento constitutivo de la estructuración económica en torno a las cadenas globales de valor-ha presentado en 2017 una contracción del 23\%. En ello, EEUU continuó siendo el principal emisor de IED, el cual en términos individuales presentó un incremento de 18\% (UNCTAD, 2017) ${ }^{16}$.

Por su parte, y acentuando la tendencia de una "hiperglobalización financiera", se han fortalecido en el período inmediato a la elección de Trump y del Brexit, las inversiones en cartera a nivel global. Conforme a la UNCTAD, los flujos de capital crecieron 1.3 puntos porcentuales del PBI global, avanzando el 6,9\% de este indicador. Los préstamos bancarios y los portafolios de inversión explican el grueso de esos flujos, en tanto que se observa un crecimiento de las deudas. En este aspecto, la globalización no presenta cambios sustantivos en su conformación. Los mercados financieros presentaron un elevado grado de interdependencia. Y como puede verse en el informe del FMI, los indicadores de tasas de interés, y expectativas en torno a tasas de políticas monetarias de las economías más avanzadas presentaron una correlación en la intensidad y en el sentido de sus variaciones. En los últimos meses, uno de los hechos más relevantes fue el aumento de los rendimientos de los bonos del tesoro estadounidenses a 10 años, los que generaron repercusiones en Alemania, Reino Unido, y Japón, entre otros; repercutiendo, asimismo, en los mercados emergentes. Estos mercados, a su vez, se ven sustancialmente afectados ante variaciones de las políticas monetarias de los países centrales, considerando particularmente los anuncios de la FED.

Por último, un dato significativo del estado de la globalización es el incremento del flujo de datos y el comercio de base tecnológica, los cuales han evolucionado de manera dinámica ${ }^{17}$. Por ejemplo, como se indica en el informe del World Economic Outlook del FMI -de abril de 2018- en 2017 los teléfonos inteligentes aportaron alrededor de un sexto de la tasa estimada de crecimiento del comercio mundial.

La evolución de la base material de la globalización, sumado a la persistencia de conductas cooperativas en las relaciones comerciales internacionales, que no involucran a los EEUU, contribuye a argumentar que se trata de una crisis en la globalización antes que una crisis de la globalización.

No obstante, la situación del orden existente es vulnerable. El contagio hacia políticas no cooperativas, la eventual salida de EEUU de la OMC -sugerida por Trump en algunas de sus comunicaciones públicas y desmentida por otros funcionarios de gobierno- y el estancamiento económico, o bien el estallido de una nueva burbuja

\footnotetext{
${ }^{16}$ Es posible sugerir que parte de esa contracción se deba a la incertidumbre de la dimensión política; Otros factores que puede considerarse es la expansión de estos indicadores en 2016 (UNCTAD 2017). Respecto de la expansión de la IED de EEUU, se especula que la misma puede deberse a una conducta de las empresas para anticiparse a la reforma fiscal.

17 Véase McKinsey Global Institute 2016
} 
financiara-dado el nivel de deuda acumulado-pueden transformar a este escenario en una crisis sistémica de la globalización.

Deben señalarse, además, otros componentes del orden liberal que han entrado en crisis: según el Índice de Democracia, producido por The Economist Intelligence Unit's, el mundo está en un declive de su apoyo a este tipo de régimen político. Ninguna región logró mejorar sus desempeños durante 2017. Se observan electorados divididos, problemas en la libertad de expresión, y una reducción en la participación popular en las elecciones, así como una menor confianza en las instituciones. Por su parte, no puede dejar de mencionarse la presencia de desafíos que implican el retiro de EEUU del Acuerdo de París, y de la UNESCO, el desfinanciamiento a Naciones Unidas, el traslado de la embajada de Israel de Tel Aviv a Jerusalén, la denuncia del acuerdo con Irán, como signos de un EEUU menos cooperativo y menos multilateralista.

\section{Reflexiones finales}

A casi dos años del inicio de esta crisis política de la globalización, predomina un clima de incertidumbre en los circuitos académicos y políticos. No obstante, la base material de la globalización continúa en expansión, aunque propio de su mismo dinamismo va expresando ajustes, tal como el paso de un énfasis en el comercio y la producción a un énfasis en los circuitos financieros. En términos de la dimensión política-ideológica-institucional, la llegada al gobierno de EEUU de la administración Trump manifiesta las transformaciones en curso, que son resultado de la misma globalización. El efecto de sus políticas, presenta desafíos sustantivos al orden existente pero no revierte el hecho de que la opción prioritaria del resto de los Estados sigue siendo la de la cooperación internacional, dentro de los marcos de gobernanza existentes. Estos elementos contribuyen a concluir que se trata de una crisis en la globalización, antes que una crisis de la globalización.

La capacidad de este análisis de sostenerse en el tiempo dependerá de la fortaleza que, frente al reto constante que supone la administración Trump, demuestren los regímenes existentes, por un lado, para mantener al resto de los Estados dentro de esferas cooperativas; y por el otro, para constreñir las opciones de Trump de acentuar sus medidas mercantilistas. En tanto que no surgen regímenes alternativos, se pone a prueba las capacidades del sistema de funcionar sin un líder o a pesar de las resistencias de su exlíder. Tal es la clave para que una crisis profunda de la dimensión política-ideológica-institucional, perfore o no la dimensión material y se transforme en una crisis sistémica de la globalización.

Recibido: 13/07/2018

Aprobado: 27/10/2018

\section{Bibliografía}


ALVAREZ GARCIA, Santiago (2018); "La reforma fiscal Trump: un comentario de sus aspectos más destacados" Cuadernos de Información Económica, n² 263, p 83-90.

BALDWIN, Richard (2016) The Great Convergence: Information, Technology and the New Globalization. Belknap Press

BOUGHTON, James; LOMBARDI, Domenico; MALKIN, Anton (2017) "The Limits of Global Economic Governance after the 2007-09 International Financial Crisis" Global Policy, 8 (4) 30-41.

COOPER, Andrew; POULIOT, Vincent (2015) "How much is global governance changing? The G20 as international practice" Cooperation and Conflict, 50 (3) 334350

DOLLAR, David (2017) "Executive summary" en WORLD BANK "Global Value Chain Development Report 2017. Measuring and Analyzing the impact of GVCs on economic development" Banco Mundial, Washington.

EUROPEAN COMMISSION (2017) "Reflection paper on Harnessing Globalization". Bruselas

FOLTEA, Marina (2018); "Options for breaking the WTO Appellate Body deadlock", ICTSD, disponible en: https://www.ictsd.org/opinion/options-for-breaking-the-wtoappellate-body-deadlock

FUKUYAMA, (2016); "US against the world? Trump's America and the new global order" Financial Times, 11/11/2016. Disponible en https://www.ft.com/content/6a43cf54-a75d-11e6-8b69-02899e8bd9d1

GARRIDO, I., MORENO, P., \& SERRA, X. (2016). "El nuevo mapa de las Instituciones Financieras Internacionales". Boletín Económico, 1/2016. 55-7

GOUREVITCH, Peter (1978); "The second image reversed: the international sources of domestic politics" International Organization, 32 (4) 881-912.

HALE, Thomas; HELD, David (2018); "Breaking the Cycle of Gridlock", Global Policy, 9 (1) $129-137$

HAYS, Jude (2017); "Embedded Liberalism and the Populist Backlash". University of Pittsburgh.

IACOVIELLO, Matteo; NAVARRO, Gaston (2018); "Foreign Effects of Higher U.S. Interest Rates" International Finance Discussion Papers 1227, Board of Governors of the Federal Reserve System (U.S.).

IKENBERRY, John (2017); "The plot against American Foreign Policy: Can the liberal order survive" Foreign Affairs, 96. p. 2-7

KEOHANE, Robert (1986); "Reciprocity in International Relations", International Organization, 40 (1) 1-27

KOTZ, David (2009); "The Financial and Economic Crisis of 2008: A Systemic Crisis of Neoliberal Capitalism", Review of Radical Political Economics, 41(3) 305-317 DOI: 10.1177/0486613409335093

LIGHTHIZER, Robert (2017) Statement by Ambassador Robert Lighhizer, United States Trade Representative. WTO. WT/MIN(17)/ST/128

MBAYE, Samba; MORENO BADIA, Marialuz; CHAE, Kyungla (2018); "Global debt database: methodology and sources" IMF Working Paper, WP/18/11 
McKinsey Global Institute (2016); Digital Globalization: The new era of global flows. McKinsey\&Company

OMC (2018); Report on G20 Trade Measures (mid-october 2017 to mid-may 2018). Disponible https://www.wto.org/english/news_e/news18_e/g20_wto_report_july18_e.pdf

PRIMO BRAGA, Carlos (2017) "The Threat of Economic Disintegration" en PRIMO BRAGA, C. y HOECKMAN, B. Future of the Global trade order. Ed.: European University Institute

RUGGIE, John (1992); "Multilateralism: the anatomy of an institution", International Organization, 46 (3) 561-598

SERBIN, Andrés (2018); "América Latina y el Caribe frente a un nuevo orden mundial: crisis de la globalización, reconfiguración global del poder y respuestas regionales", en SERBIN, Andres (ed), América Latina y el Caribe frente a un Nuevo Orden Mundial: poder, globalización y respuestas regionales. Buenos Aires, Ediciones CRIES.

STIGLITZ, J. E. (2002). Globalization and its Discontents, New York: Norton.

SUBRAMANIAN, Arvin; KESSLER, Marin (2013); "The hyperglobalization of trade and its future". Peterson Institute for International Economics, Working Paper Series, WP13-6.

UNCTAD (2017). "Tendencias y retos actuales de la economía global" en Informe sobre el Comercio y el Desarrollo 2017. Ginebra: United Nations Publications.

VANGRASSTEK, Craig (2017); "Back to the Future: US Trade Policy under the Trump Administration" en PRIMO BRAGA, C. y HOECKMAN, B. Future of the Global trade order. Ed.: European University Institute WALLERSTEIN, I. M. (1979). "EI moderno sistema mundial. La agricultura capitalista y los orígenes de la economía-mundo europea en el siglo XVII". Revista de estudios políticos, (12), 223-225.

WALLERSTEIN, Immanuel (1997); "La reestructuración capitalista y el Sistemamundo", Conferencia magistral en el XX⿳ Congreso de la Asociación Latinoamericana de Sociología, México, 2 al 6 de octubre de 1995

WOODS, Ngaire (2010); "Global Governance after the Financial Crisis: A New Multilateralism or the Last Gasp of the Great Powers?" Global Policy, 1 (1) 51-63.

ZURN, Michael (2018), "Contested Global Governance”, Global Policy, 9 (1). 138-145 